\title{
The Application of Intelligent Voice Analysis Technology in the Customer Call Center of Telecom Operators
}

\author{
Zhang Kai, He Jin, Yang Jinzhou, Zhang Xiao, Wang zhijun \\ Platform and Production Laboratory \\ China Unicom Institute \\ Beijing, P.R.China \\ zhangkai0613@sina.com
}

Keywords: Intelligent voice recognition; Telecom operators; Customer service center; Cloud computing

\begin{abstract}
Call center is one of the most important means for telecom operators to provide after-sales service for customers. Every day, call center can produce a large number of voice data. But these data do not bring significant value to the enterprise. Recent years, the development of intelligent voice technology has brought a turning point for the operator call center. By using the intelligent voice technology, telecom operators can analyze user behavior characteristics. And then they can develop a behavioral warning, precision marketing and other business strategies. In this article, we introduce the key technologies of intelligent voice technology which could be used in call center. And base on the cloud computing technology, we point out the overall architecture and working mechanism of the intelligent voice analysis system. The new system architecture will bring great value to telecom operators.
\end{abstract}

\section{Introduction}

Customer service hotline of telecom operators is one of the most important channels for customer contacting with the company. It is an important way to provide after-sales support and maintain the relationship between the company and customers. It is also an important source of customer-related information, content of services and quality of information. With the continuous development of information technology, customer service call center system had undergone a series of changes, as shown in Fig1. In the first generation, voice traffic access (voice card) was appeared. In the second generation, CTI technology aimed at combining talk with business. In the third generation, multi center net call was realized. In the fourth generation, IPCC technology which can support flexible networking agents, access equipment and multimedia service was appeared. It is now currently developing to the fifth generation of internet customer service call center. The so-called internet customer service is a comprehensive combination between traditional call center and the new internet technology. So that the customer service system ability can get a substantial upgrade, result in a great value for the enterprise. Among them, the emergence and improvement of the intelligent voice analysis technology brings the unprecedented opportunity for the service center[1-2].

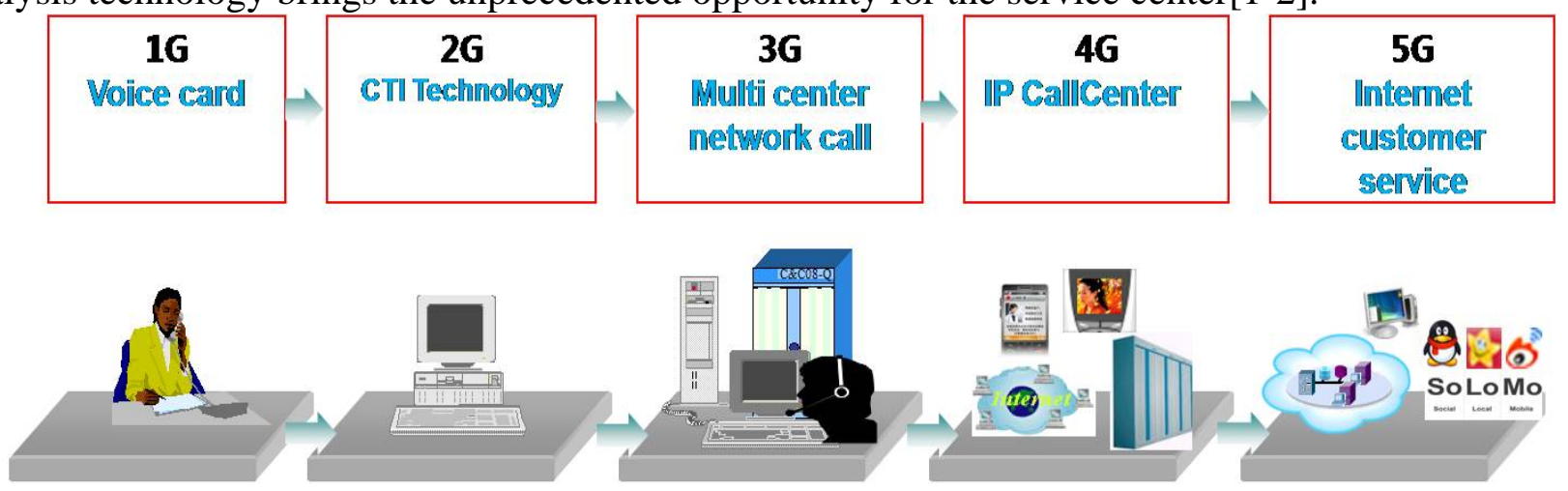

Fig. 1. Development of telecom operators call center 
Combined with the technology of intelligent speech analysis, it can bring many benefits for telecom operators[3-4].

\section{Intelligent Voice Analysis Technology}

Voice recognition is a technology to realize that the machine could understand human language. By the content of identify, voice recognition technology can be divided into semantic recognition and acoustic recognition. The purpose of semantic recognition is to convert lexical content of human speech into the processed input of the computer. Acoustic recognition mainly refers to the voiceprint recognition technology, which can extract speaker's unique acoustic features from the speech signal, and then identify the speaker's identity. Voice recognition is to make the right response to the request and the inquiry contained in the oral language, but not limited to the correct conversion of all words[5-6].

The whole process of voice analysis should have the following key steps:

Voice transcription

Voice transcription is the most important step in voice analysis. It can establish a text index and convert the non structured audio file into the structured text information.

Voice analysis system firstly converts separated speech into the Chinese phonetic symbols by acoustic transformation model. And then phonetic information can be corresponded to the text content by the help of large vocabulary network language model. In this process, we need to optimize the acoustic model by considering local dialect.

Establish index

Voice analysis system can detect a certain period of fundamental frequency and high frequency in a telephone recording. So it could recognize the position of the mood swings in the entire speech, average talking speed, talking speed changing and the time that users and service personal both are not speak. All of the information are eventually changed into index files for the standard XML format.

Voice retrieval

Voice retrieval rapidly screens the keywords information of emotional detection information and mute information from all of the index files and returns the concerned voices. The core of voice retrieval is mainly including key words, abnormal emotion and the long mute time.

Scene segmentation

In the current hotline platform, system records all the dialogues of both the customer service staffs and customers at the same time. During the voice analysis process, we need to separate the two parties. On one hand, we can monitor customer service quality and improve customer service level. On the other hand, we can analyze the hot issues, suggestions and opinions in the user's speech.

Cluster service

Customer service hotline will generate more and more massive data. Single server can not deal with the massive amounts of voice. So voice analysis system needs to support cluster service. When the call amount is increasing, the service ability can be improved by adding more servers.

\section{Problems of current customer service center}

Quality testing

Call center is an important voice service window of telecom operators. Every day, hundreds of artificial service call recording are preserved. But at present, only $1 \%$ to $3 \%$ of the audio file is used to manually inspection. Most of the hidden value information in speech files are not effective mining. Mainly in the following several aspects:

The extraction strategy of recording is single. So it can not reflect the rich features of the dialogues, such as the user's emotional expression and the calling key words.

The amount and the efficiency of manual listening is extremely low. So the huge numbers of valuable information in the recording files are ignored. 
Only focus on the analyses of the operator service quality level, and ignore customer demands hidden in the dialogues.

User satisfaction

In the twenty-first century when business competitions are increasingly fierce, improving customer satisfaction and strengthening customer loyalty are becoming hot topics. Among them, the user experience is particularly important. Suppose a situation that a customer calls to a call center to consult a problem. But the result of the call is "the seat are busy now, please wait patiently". After a long waiting, the seating member answers the phone. Then the seating member says "sorry, I cannot solve this problem, please wait a moment, I will help you transfer another expert" as the user showing his description. So the user has to describe the problem to another expert again. We can imagine the user's patience is gradually disappearing so as the customer satisfaction. When a sudden situation appears, a huge numbers of users have to call the center call simultaneously. This will result in the call blocking. Telecom operators often increase and improve the processing capacity to protect the business all right. But it is not a wise choice.

One of the telecom companies has encountered such a situation. The administrator suddenly found that the calling amount was far beyond the normal value in that day. They didn't know what happened and cannot listen to the recording of all that day because the calling amount is very large. At that moment, the voice analysis system helped them a lot. By using the intelligent voice analysis, they found the real reason of that problem from the key words in the huge amount of callings. Several days ago, the telecom company launched a new mobile phone business and commit that the phone will be sent to the hands of customers in three days. But in fact, due to logistics and other reasons, customers in some areas needed to wait five days in order to receive. So customers had to call to ask the reason in the sixth day. That resulted in the call amount sharply increased in that day. Not only made the seating members continuously to answer the call to the customer, but also caused the call congestion. Finally, it affected the normal business management. By finding the reason of the problem in time, the company took the measures and call amount decreased to the average value quickly.

\section{Intelligent voice system architecture}

There are three main layers in an advanced IT architecture, as shown in Fig.2.

IaaS(Infrastructure-as-a-Service): The IaaS provides on-demand resource services, including computing, storage and networking and other basic computing resources delivery and management capabilities. Users can deploy any software on the above.

PaaS(Platform-as-a-Service): The PaaS provide on-demand automatic platform services, including API, tools and services to deploy and manage applications and services for users. Services mainly provided for the testers, IT operator and IT manager.

SaaS(Software-as-a-Service) : Based on scalable cloud architecture, the SaaS provides on-demand applications, such as office applications, digital business acceptance, service applications, etc. Based on the background of standardized service capabilities, the front desk flexible configuration, assembly and provide personalized services. 


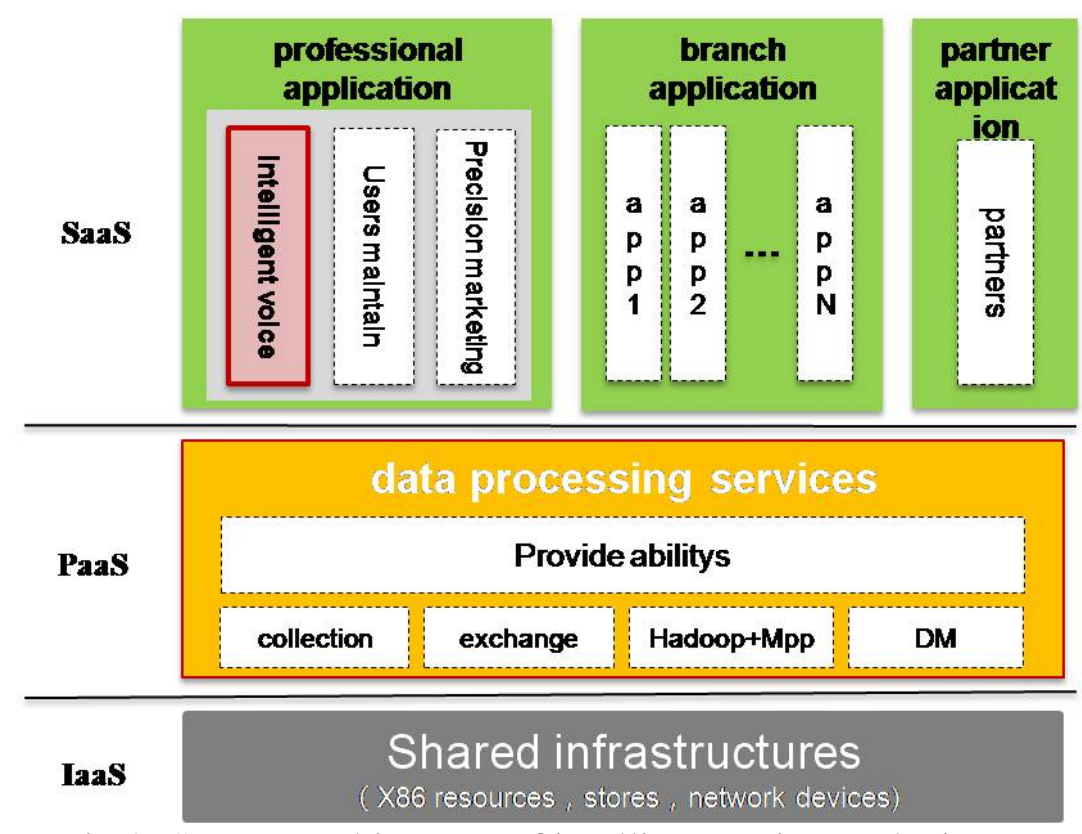

Fig.2. System architecture of intelligent voice analysis

The previous paragraphs talked about the cloud architecture. We advice that the intelligent voice analysis system should be an innovative application in the SaaS layer based on big data platform(PaaS). And in our architecture, intelligent voice analysis is mainly composed of three parts: recording file collection, recording and translation and data analysis, as shown in Fig.3:

The first step is the recording file collection. The system collects hotline service record files from call center recording servers. And also collects service request data from customer service business systems.

The second step is the recording translation. According to the language models and acoustic models of the telecommunications industry and using the technology of voice recognition and semantic understanding, non structured audio files will be translated into structured texts. Finally, system could set up a structured recording database.

The third step is data analysis. We need to set up many business models and quality testing models for different application levels and continuously optimize them. By using these models, we can cross analyze and hot spot analyze the customer callings in different dimensions. According to the business requests to specially analyze, and enhance the service level.

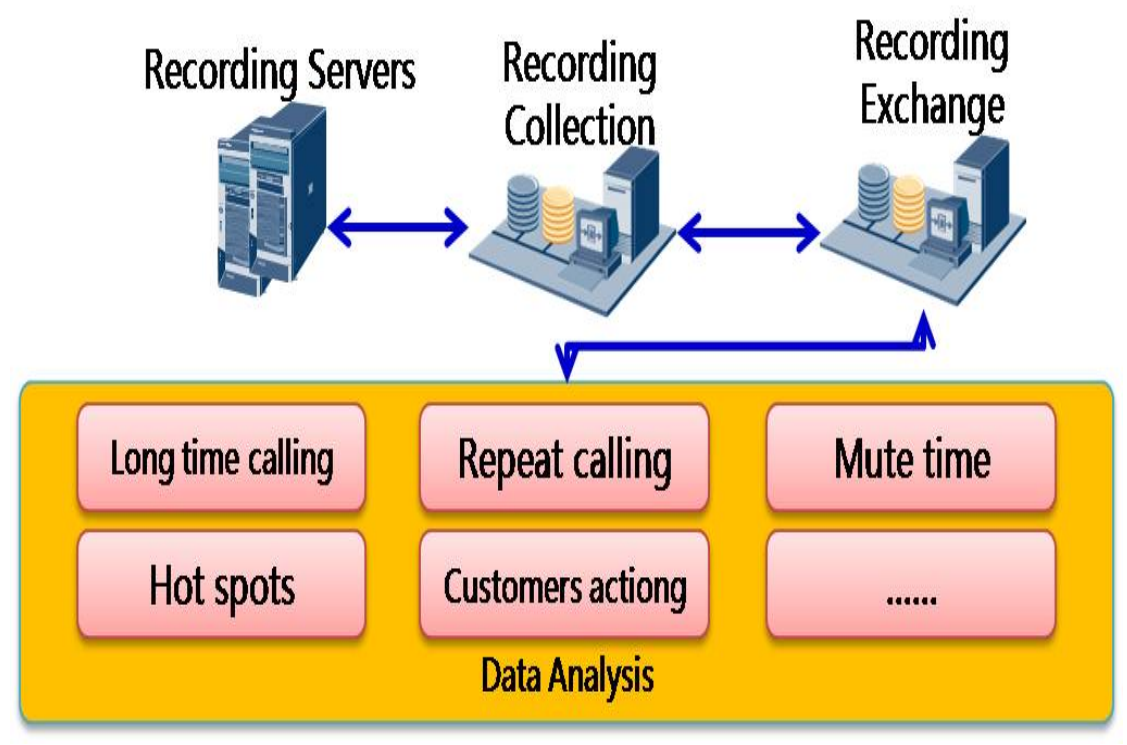

Fig.3. Key technologys of intelligent voice analysis

Fig.4 shows overall scheme of intelligent voice analysis system. The system can be divides into several parts: data collection, recording files, exchanged text and data analysis as discussed in the preceding paragraphs. 


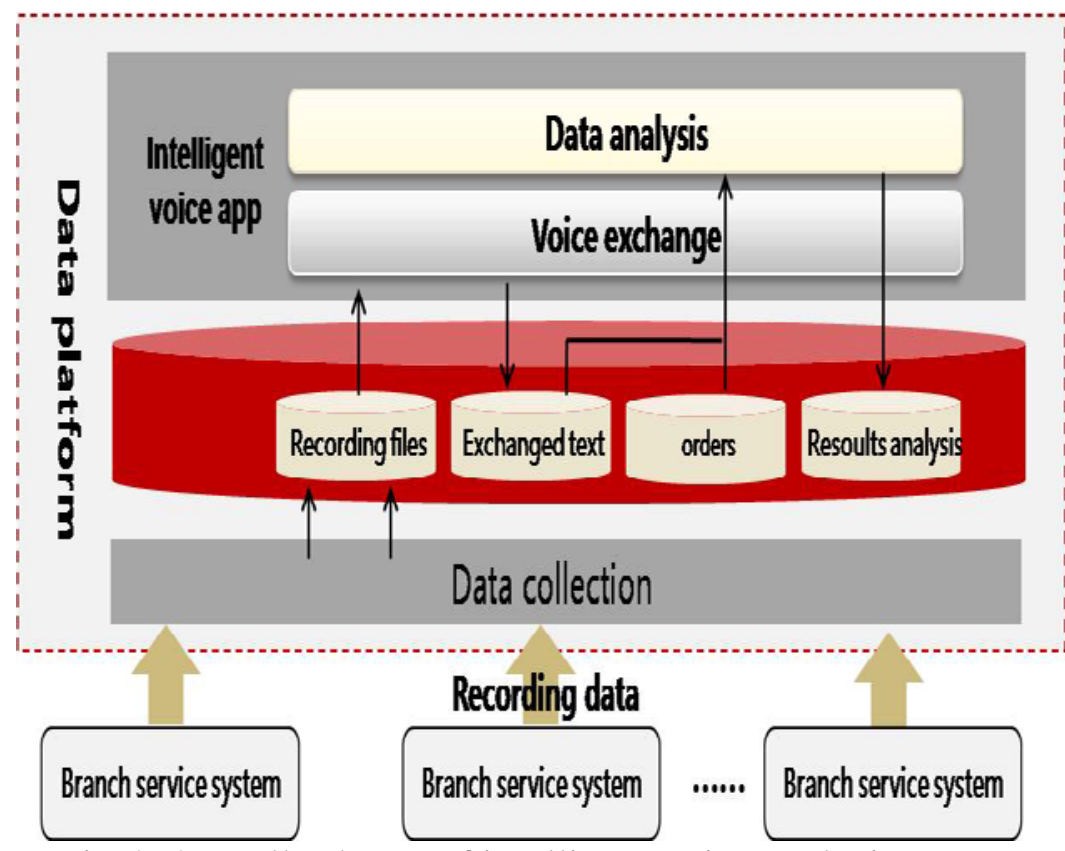

Fig.4. Overall scheme of intelligent voice analysis system

The provincial branch companies are responsible for collecting the recording and customer contact and service request records by unified specification. And also is the responsible for managing, outputting these data files.

The unified big data platform is responsible for the processing of data. The big data platform firstly exchanges the huge amount of voice data into standard voice files. Then these standard XML voice files can be analyzed by using the model which we have prepared before. After these processes, the analysis results will be sent to the intelligent voice applications, and exhibit to the staffs to use. Finally, all of the standard XML voice files should be stored by using unified specification, and also the results should be stored in the big data platform.

\section{Summary}

Call center is one of the most important means for telecom operators to provide after-sales service for customers. Every day, call center can produce a large number of voice data. But these data do not bring significant value to the enterprise. Recent years, the development of intelligent voice technology has brought a turning point for the operator call center. By using the intelligent voice technology, telecom operators can analyze user behavior characteristics. And then they can develop a behavioral warning, precision marketing and other business strategies. In this article, we introduce the key technologies of intelligent voice technology which could be used in call center. And base on the cloud computing technology, we point out the overall architecture and working mechanism of the intelligent voice analysis system.

\section{References}

[1] Kreiman Jody, Antoñanzas-Barroso Norma, Gerratt Bruce R. Integrated software for analysis and synthesis of voice quality.[J]. Behavior Research Methods (Online), 2010, 42(4).

[2] A. I. Mekhannikov, V. A. Perepelkin. Synthesis of transmission receiving transducers for multipurpose radio standards in the microwave band[J]. Measurement Techniques, 1997, 40(4).

[3] Albertini G, Bonassi S, Dall'Armi V et al.. Spectral analysis of the voice in Down Syndrome.[J]. Research in Developmental Disabilities, 2010, 31(5).

[4] Latinus Marianne, Belin Pascal. Anti-voice adaptation suggests prototype-based coding of voice identity.[J]. Frontiers in Psychology, 2011, 2.

[5] Zhu Jinzhou, Li Aimin, The trends of call center [J] China Science and Technology Expo, 2012(9) 
[6] Zhang Rong. Design and implementation of CTI-based call center technology [D] Xi'an University of Electronic Science and Technology,2009 